\title{
Adaptive optical satellite network architecture
}

Hideaki Kotake, Yuma Abe, Tetsuharu Fuse, Toshihiro Kubooka, Morio Toyoshima

Hideaki Kotake, Yuma Abe, Tetsuharu Fuse, Toshihiro Kubooka, Morio Toyoshima, "Adaptive optical satellite network architecture," Proc. SPIE 11852, International Conference on Space Optics - ICSO 2020, 118521M (11 June 2021); doi: 10.1117/12.2599250

SPIE Event: International Conference on Space Optics - ICSO 2021, 2021, Online Only 


\section{International Conference on Space Optics-ICSO 2020}

Virtual Conference

30 March-2 April 2021

Edited by Bruno Cugny, Zoran Sodnik, and Nikos Karafolas
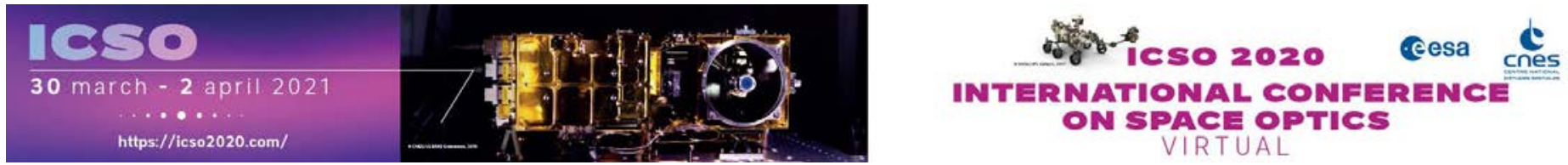

\section{Adaptive optical satellite network architecture}

\section{Cesa isopmeatians ecnes}




\title{
Adaptive Optical Satellite Network Architecture
}

\author{
Hideaki Kotake*, Yuma Abe, Tetsuharu Fuse, Toshihiro Kubooka and Morio Toyoshima \\ National Institute of Information and Communications Technology, \\ 4-2-1 Nukui-Kitamachi, Koganei, Tokyo, 184-8795, Japan
}

\begin{abstract}
An adaptive optical satellite network (AOSN) for next-generation optical satellite network systems is proposed. To decrease operational cost of complicated network systems, the AOSN can monitor status of satellite optical links and modify the link parameters of laser-communication terminals (LCTs) automatically. To realize the AOSN, we utilize adaptive optical transmitters, digital coherent optical receivers and software defined network (SDN) and incorporate them into a conventional concept of adaptive optical networks. The AOSN can realize the adaptive control of link parameters for a variety of satellite optical links with the utilization of artificial intelligence (AI), machine learning and operation policy.
\end{abstract}

Keywords: Adaptive Optical Satellite Network (AOSN), Optical satellite communications, Adaptive optical transmitters, Digital coherent optical receivers, Software Defined Network (SDN)

\section{INTRODUCTION}

High data-rate satellite communications have been on the rise due to the emergence of Earth-observation (EO) services and satellite Internet services [1]. Optical satellite communication is a promising technology to deliver a large volume of data in the space environment. Research and development for optical satellite communications have been performed worldwide. The 1.8-Gbit/s data relay service has been already started by the European data relay system (EDRS) between geostationary Earth orbit (GEO) satellites and low Earth orbit (LEO) satellites [2]. The National Aeronautics and Space Administration (NASA) will launch the Laser Communication Relay Demonstration (LCRD) satellite into GEO in 2021, and will demonstrate 2.88-Gbit/s optical satellite communications [3]. The Japan Aerospace Exploration Agency (JAXA) launched the Optical Data Relay Satellite (ODRS) into GEO in 2020 and optical data transmission between a GEO satellite and the LEO satellites will be demonstrated [4]. The Engineering Test Satellite 9 (ETS-9) will be launched to verify 10-Gbit/s optical feeder link (HICALI) between ground and GEO in both directions [5]. Spacegrade design and verification of the transponder for an optical feeder link have been investigated [6]. The Small Optical TrAnsponder (SOTA) [7] mission successfully demonstrated a miniaturized terminal onboard a microsatellite and the Very-Small Optical Transmitter (VSOTA) [8] was launched as an optical link demonstration between a LEO microsatellite and ground. Demonstrations for LEO-to-GEO and LEO-to-ground links using CubeSats are planned in the CubeSOTA mission [9]. SONY Computer Science Laboratories (CSL) has also carried out the SOLISS (Small Optical Link for International Space Station) mission [10,11]. Intersatellite laser-communication terminals (LCT) for LEO-toLEO optical satellite links have been developed by Mynaric [12]. These approaches are accelerating the realization of various applications such as distribution of sensing data obtained by satellites and satellite Internet services.

Under this scenarios, several types of satellite optical-communication links such as GEO-to-LEO, LEO-to-LEO, groundto-GEO, and ground-to-LEO will be operated as practical optical satellite-communication systems in the near future. Various types of optical modulation and detection schemes and data rates will be used in such the systems. It has been considered that system operations for optical satellite links are being more complicated and operational expenses (OPEX) are rapidly increasing in order to manage a variety of links and optical signals, which will exist in satellite optical network systems. Currently, telework is being established worldwide for the infection prevention of the COVID19 virus. After containing the present pandemic situations, many companies and institutions have announced to continue promoting telework. As a consequence, admissions for system operation offices have strongly being restricted. It has been expected that the number of system operation staffs will decrease in the near future. We consider that nextgeneration satellite optical-communication system will be necessary to solve these problems. 
The Adaptive Optical Network (AON) such as the elastic optical network (EON) has been studied in terrestrial wavelength division multiplexing (WDM) optical networks [13-15]. AON is a concept of optical fiber network systems, which monitors link status and modify the link parameters of optical nodes. In addition, system operation and maintenance have been performed automatically. In this paper, the Adaptive Optical Satellite Network (AOSN) which applies AON concept for next-generation optical satellite network system is proposed. Our proposed system monitors status of optical satellite links and modify the parameters of LCTs automatically. Furthermore, adaptive routing and control of link parameters for a variety of optical satellite links can be realized using network controllers.

This paper is organized as follows. The system concept of the proposed AOSN architecture and related researches for the AOSN is described in Section 2. System requirements of the AOSN are described in Section 3. The system architecture of the AOSN is described in Section 4. The conclusions are described in Section 5.

\section{SYSTEM CONCEPT}

\subsection{Related research}

System-operation staffs manage each optical node via network management system (NMS) in the conventional terrestrial WDM optical networks. They operate the optical networks by the following procedure: collect the performance and link status information monitored by optical nodes, analyze the link-status information, decide the routing details and link parameters of optical nodes, and control them in the optical networks. The link-status information is described as follows: bit error rate (BER), received optical channel power, linear impairments, and nonlinear impairments. The link parameters are also described as follows: fiber routes, modulation formats, data rate, number of optical channels and optical channel power. However, as the number of optical nodes increases, it is concerned about dramatic increase of OPEX including handling for increase of communication demands, accidents and maintenances. It is certainly possible that system operation staffs cannot handle all these system operations in the near future.

The AON concept has been proposed and studied in optical-fiber communication area such as EON to solve the above problems [13-15]. The AON is a concept of an optical-fiber network, which manages system operations and maintenances automatically instead of system-operation staffs. AON conducts the operating procedure of collecting, analyzing, deciding and controlling an optical-fiber network automatically by NMS, which adopts artificial intelligence (AI), machine learning, and operational policy.

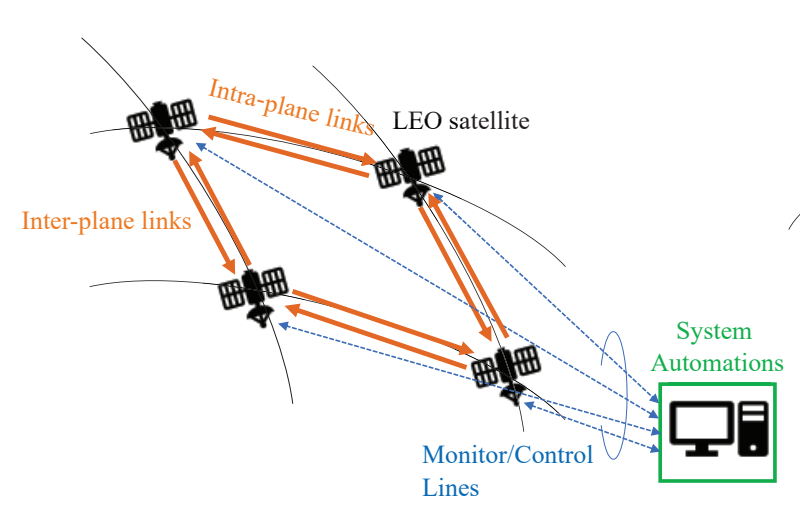

(a)

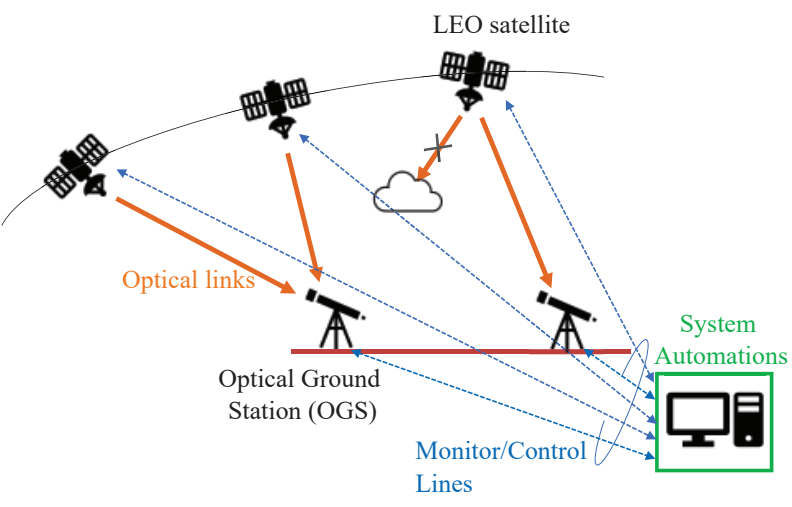

(b)

Figure 1. Concept of the adaptive optical satellite network (AOSN) (a) LEO-to-LEO satellite configuration, and (b) groundto-LEO satellite configuration.

\subsection{System concept}

It has been considered that the increase of OPEX and decrease of number of system operation staffs, as shown in Subsection 2.1, will clearly appear in satellite optical networks. Therefore, we need the AOSN which utilizes the AON 
concept to operate satellite optical networks automatically. Figure 1 shows a concept of the AOSN. The AOSN operates the optical networks automatically by the following procedure: collect the link status information of optical satellite links, analyze the link status information, decide the routing details and link parameters of LCT, and control the routing details and link parameters. The above procedure is conducted automatically by network controllers, which adopt AI, machine learning and operational policy. LCTs include optical transmitters, receivers, amplifiers, optical assemblies, and they are implemented in satellites and optical ground stations (OGSs). AOSN flexibly establishes the connections, depending on link status, by controlling the link parameters of LCTs.

\section{SYSTEM REQUIREMENTS}

There are three main functions such as the flexible optical transceiver function, the link-status monitoring function, and the flexible control function of link parameters to realize the AOSN. Three main functions to be implemented in the AOSN are described as the system requirements.

\subsection{Flexible optical transceiver function}

Flexible optical transceivers [16] shall transmit and receive adaptive modulation waveforms, depending on link status in the AOSN. Figure 2 shows the configuration of flexible optical transceivers. Optical transmitters need to generate optical signals employing multi-level modulation formats and symbol rates. Adaptive optical transmitters, which implements IQ modulators, two digital-to-analogue converters (DAC) and Tx digital signal processing (DSP) circuits, are the solution for the AOSN [17]. IQ modulators, including two mach Zehnder modulators (MZMs) and $\pi / 2$ phase shifters, can output optical signals employing multi-level modulation formats such as multi-level phase-shift keying (M-PSK) signals and multi-level quadrature amplitude modulation (M-QAM) signals. Multi-level modulation electrical waveforms need to be generated by high-speed electronics including Tx DSP circuit and DACs. Therefore, adaptive optical transmitters enable flexibly switching for various modulation formats of optical signals, depending on link status.

Optical receivers also need to detect optical signals employing multi-level modulation formats and symbol rates. Digital coherent optical receivers, including coherent front-end, two analogue-to-digital converters (ADC) and Rx DSP circuits, are the solution for the AOSN [18]. Coherent front-end, including 90-degree optical hybrids and balanced photo detectors (BPDs), can detect electric field of optical signal, using laser diodes (LD) as a local oscillator and ADCs. Multi-level modulation electrical waveforms can be processed by Rx DSP in the digital domain.

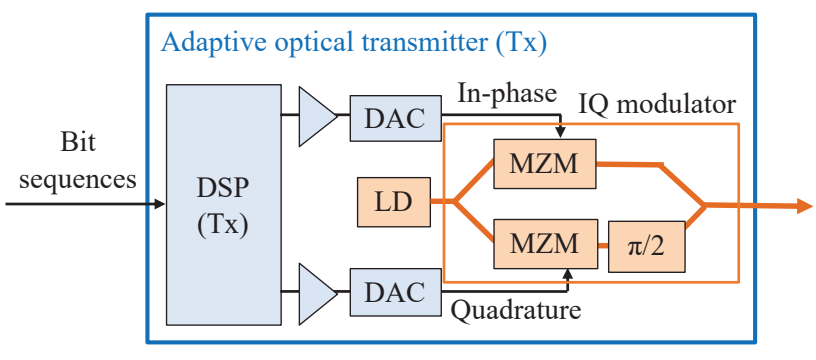

(a)

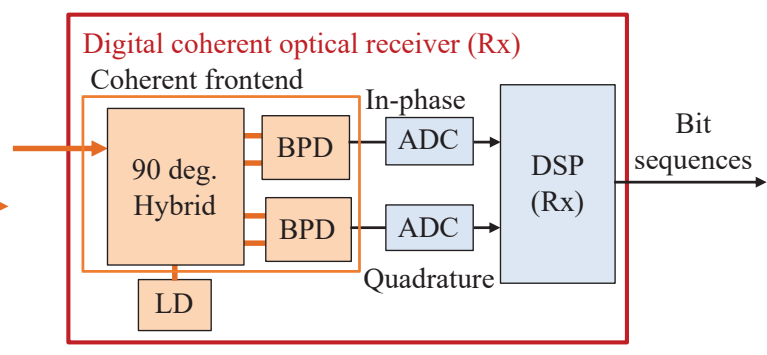

(b)

Figure 2. Configuration of the flexible optical transceiver (a) transmitter and (b) receiver.

\subsection{Link-status monitoring function}

Link-status monitoring functions are required in the AOSN in order to collect status information for optical satellite links. As digital coherent optical receiver can compensate for various impairments and can estimate status information by its own DSP circuit [19], this receiver is the solution for link status monitoring function in the AOSN. Figure 3 shows the configuration of the digital coherent optical receiver.

Several impairments, which include frequency fluctuations, power fluctuations and phase fluctuations, occurred in optical satellite links. Firstly, the Doppler frequency is considered as an example of frequency fluctuations. According to 
[20], the frequency estimation function can detect the Doppler frequency and can monitor it. Secondly, the atmospheric turbulence is considered as an example of power fluctuation. The adaptive equalization function enables monitoring power fluctuations by detecting electrical signal-to-noise ratio (SNR) after adaptive equalization [21]. Finally, nonlinear impairments occurred inside the booster amplifier [22]. The phase-estimation function can detect nonlinear phase noise and can monitor it [21].

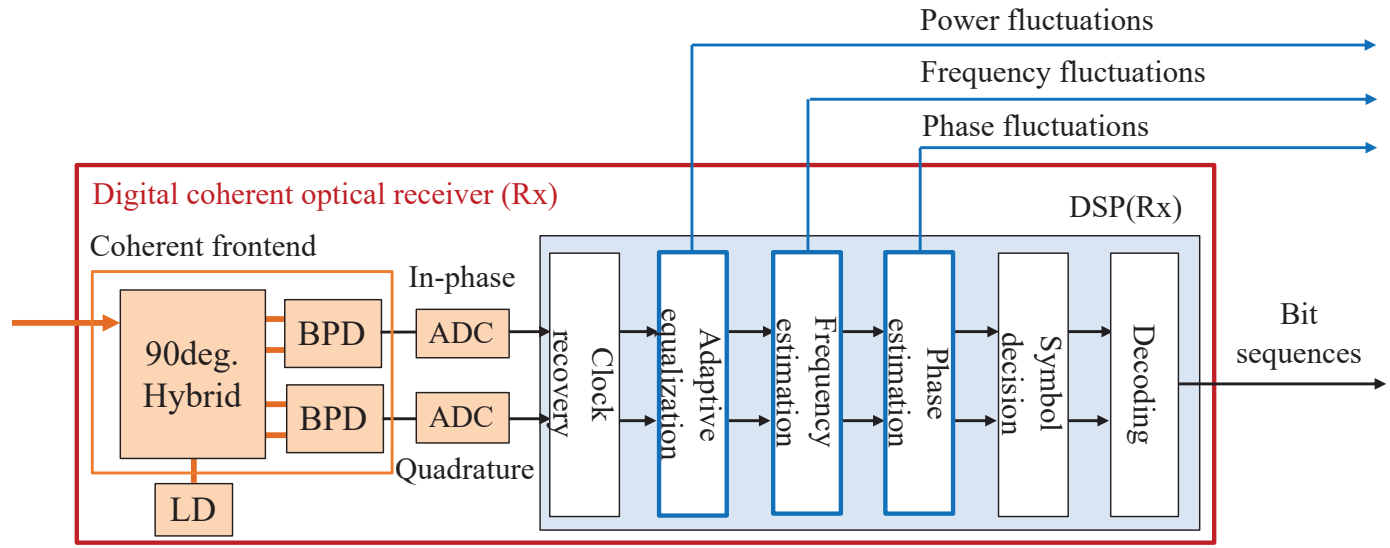

Figure 3. Configuration of the digital coherent optical receiver.

\subsection{Flexible-control function of link parameters}

Link parameters of LCTs flexibly need to be changed in the AOSN. However, as the controllers are implemented inside the components of the LCT, the network operation and configuration will become more complicated. Software Defined Network (SDN) is the solution to separate software (control) function and hardware function [23]. Figure 4 shows configuration of flexible control function of link parameters. Link parameters can be flexibly changed via SDN controllers. SDN enables the uniform management of the components of the LCT and realize network virtualization and operational efficiency. Link parameters of the LCT are described as follows: modulation formats, wavelength of LDs, number of channels, data rates, and optical output power of the booster amplifiers.

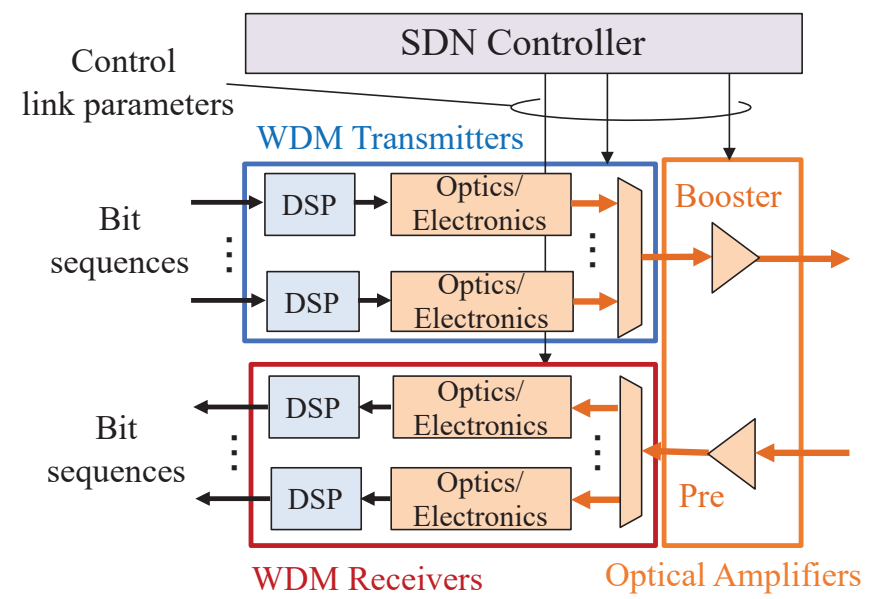

Figure 4. Configuration of the flexible-control function of link parameters. 


\section{SYSTEM ARCHITECTURE}

\subsection{System configuration}

Figure 5 shows system configuration of the AOSN for a variety of optical satellite links. As an example of AOSN, GEOto-LEO, LEO-to-LEO, ground-to-GEO, and ground-to-LEO are shown in Figure 5, LCTs for various satellite optical links are implemented in satellites and OGSs. It has been considered that a few LCTs are implemented in satellites, depending on configurations and purposes for a variety of optical satellite links. For example, in the case of LEO-to-LEO optical links, satellites may need to implement more than four LCT in order to establish four directional links between satellites.

The LCTs utilize link-status monitoring function and flexible-control function of link parameters. Link-status information monitored inside the LCTs will be collected by network controllers, which are implemented on the ground. Link parameters of the LCTs will also be decided and controlled by network controllers, which implement the SDN controllers. Adaptive controls of link parameters can be realized, depending on the link status for a variety of optical satellite links by AI, machine learning, and operational policy. The network controllers collect link-status information and control-link parameters, via radio-frequency (RF) feeder links for satellites and via ground networks for OGS, respectively.

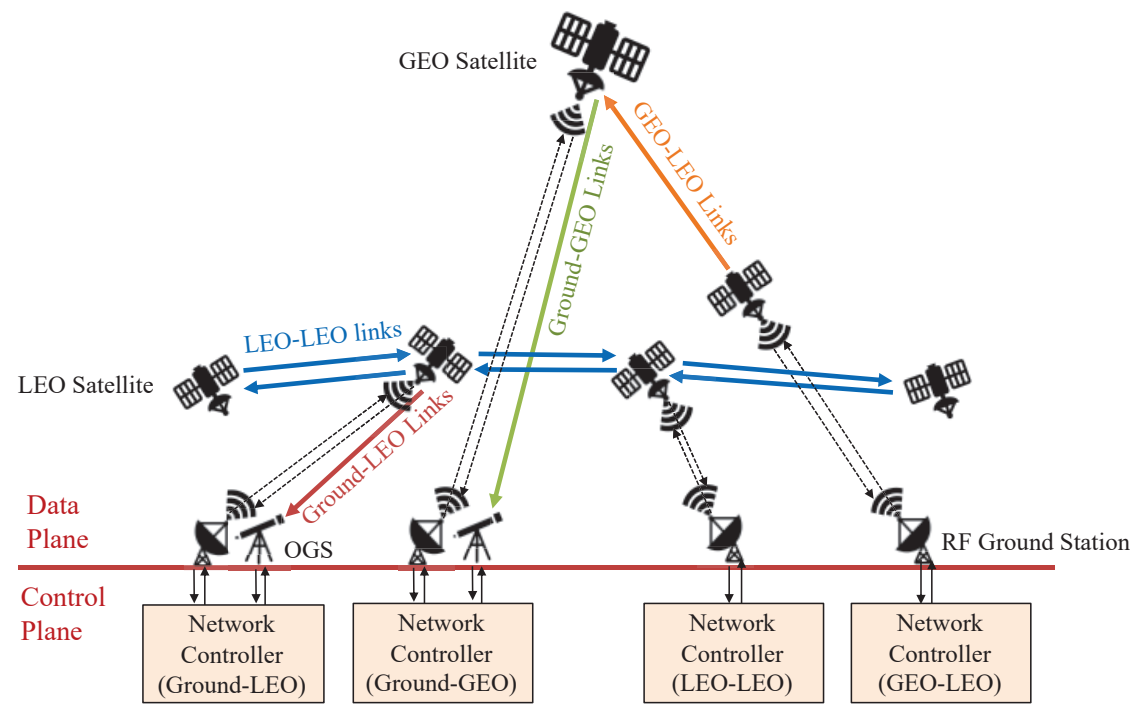

Figure 5. System configuration of the adaptive optical satellite network (AOSN).

\subsection{Configuration of laser-communication terminals (LCT)}

Figure 6 shows configuration of LCTs for the AOSN. The LCTs are implemented in satellites and OGSs and establish various optical satellite links with other LCTs. They are composed of WDM transmitters, WDM receivers, optical amplifiers, optical assemblies, data processors and terminal controllers.

WDM transmitters and WDM receivers transmit and receive WDM optical signals, which includes several optical signals, respectively. Optical amplifiers are composed of booster amplifiers and low-noise preamplifiers. The booster amplifier and the preamplifier amplify WDM optical signals on the transmitter and receiver side, respectively. Configuration of optical amplifiers shall be changed for various optical satellite links by increasing number of amplifier stages [24]. Furthermore, the optical output powers for the booster and preamplifiers can be flexibly changed, depending on the number of channels. The optical assembly conducts acquisition, tracking and pointing of WDM optical signals with other LCTs. The data processor conducts data processing. The terminal controller collects link-status information, and sets link parameters into the LCT. 


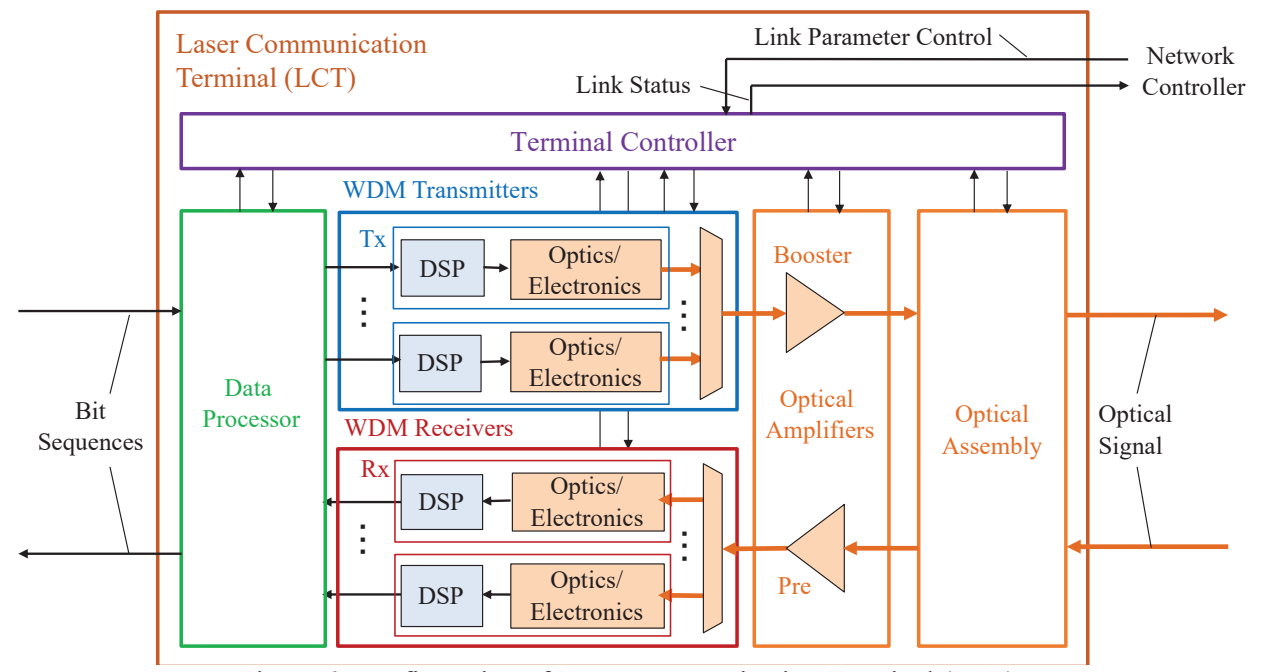

Figure 6. Configuration of Laser Communication Terminal (LCT).

\subsection{Configuration of transmitters and receivers}

Figure 7 shows configuration of WDM transmitters and WDM receivers for AOSN. WDM transmitters are composed of adaptive optical transmitters and WDM filters. WDM receivers are composed of digital coherent optical receivers and WDM filters. Adaptive optical transmitters utilize the adaptive-modulation function, the variable data-rate function and the tunable LD wavelength function. Digital coherent optical receivers utilize the digital coherent-reception function, the variable data-rate function, the tunable LD wavelength function and the link-status monitoring function. The receivers detect the link-status information, including frequency fluctuations, power fluctuations, phase fluctuations.

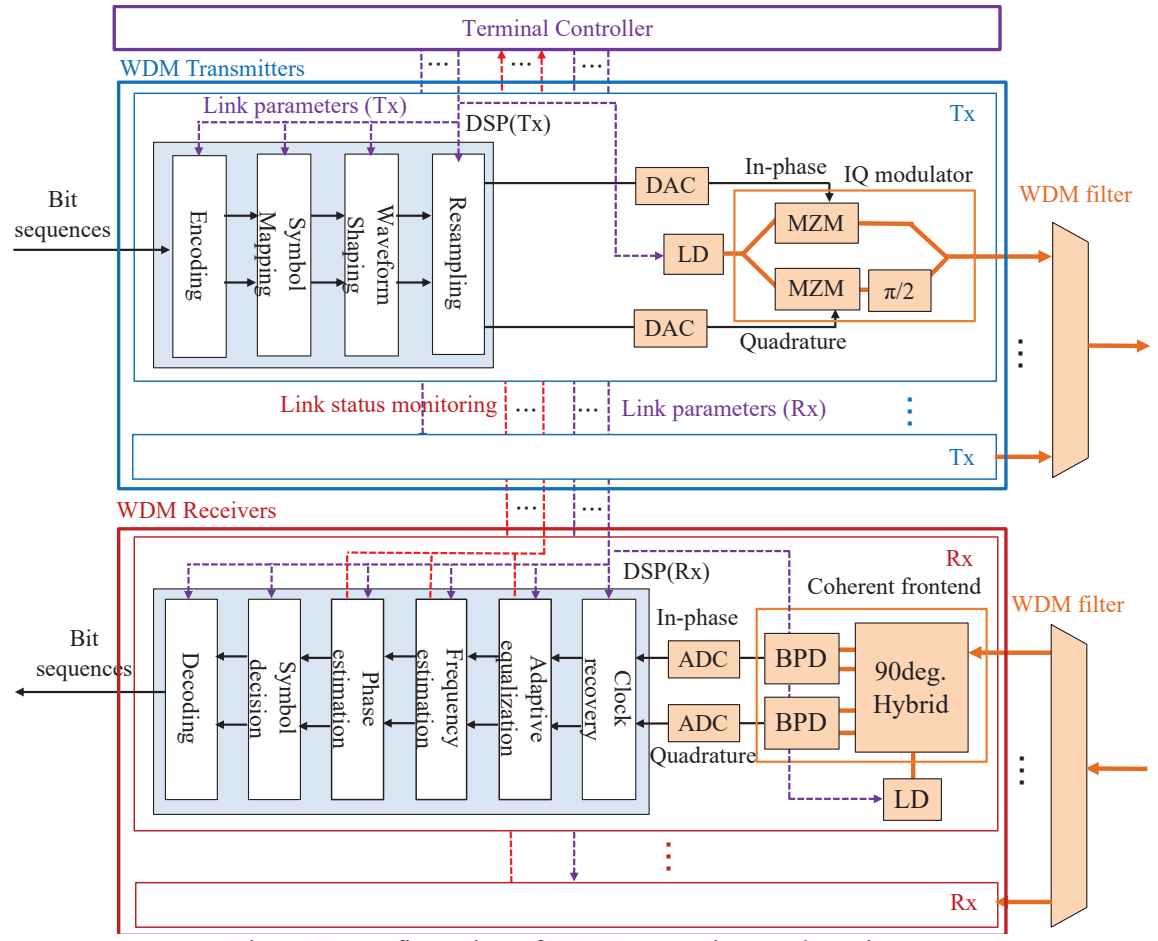

Figure 7. Configuration of WDM transmitter and receiver. 


\section{CONCLUSIONS}

The adaptive optical satellite network (AOSN) is proposed, which utilizes concept of adaptive optical network. Three main functions of AOSN, such as the flexible optical-transceiver function, the link-status monitoring function and the flexibly-control function of link parameters are presented. We have utilized adaptive optical transmitters, digital coherent optical receivers and software defined network (SDN) to realize the AOSN. We have also described the system architecture of the AOSN, which includes the system configuration, the configuration of LCTs, and the transmitters and receivers. We believe that the AOSN will become the next-generation optical satellite network system. In future works, we plan to evaluate and improve the system performance of the AOSN.

\section{REFERENCES}

[1] M. Toyoshima, "Recent Trends in Space Laser Communications for Small Satellites and Constellations," IEEE J. Lightwave Tech., vol. 39, No.3, pp.693-699 (2021).

[2] F. Heine et al., "Status of Tesat laser communication activities," Proc. SPIE, 1127204 (2020).

[3] B. L. Edwards, et al., "An overview of NASA's latest efforts in optical communications," IEEE International Conference on Space Optical Systems and applications (ICSOS), pp.1-8 (2015).

[4] S. Yamakawa, et al., "JAXA's Optical Data Relay Satellite Programme," IEEE International Conference on Space Optical Systems and applications (ICSOS), pp.1-3 (2015).

[5] Y. Munemasa, et al., "Design status of the development for a GEO-to-ground optical feeder link, HICALI," Proc. SPIE, 105240F (2018).

[6] H. Kotake, et al., "Design and verification of a space-grade $10 \mathrm{Gbit} / \mathrm{s}$ high-speed transponder for an optical feeder link," Proc. SPIE, 1091012 (2019).

[7] H. Takenaka et al., "In-orbit verification of small optical transponder (SOTA): evaluation of satellite-to-ground laser communication links," Proc. SPIE, 973903 (2016).

[8] H. Kunimori et al., "Preliminary Results of Very Small Optical Transmitter (VSOTA) on Microsatellite RISESAT," IEEE International Conference on Space Optical Systems and applications (ICSOS), S4.6 (2019).

[9] A. Carrasco-Casado, et al., "Intersatellite-link demonstration mission between CubeSOTA (LEO CubeSat) and ETS9-HICALI (GEO satellite)," IEEE International Conference on Space Optical Systems and applications (ICSOS), S4.3 (2019).

[10] K. Iwamoto, et al., "Small laser communication terminal (SOLISS) demonstration on the ISS exposure facility," IEEE International Conference on Space Optical Systems and applications (ICSOS), S4.2 (2019).

[11] H. Komatsu et al., "In-orbit experimental architecture design of bi-directional communication with a small optical communication terminal attached on ISS and an optical ground station," Proc. SPIE, 112720C (2020).

[12] C. Carrizo et al., "Optical inter-satellite link terminals for next generation satellite constellations," Proc. SPIE, $1127203(2020)$.

[13] M. Jinno, et al., "Virtualization in optical networks from network level to hardware level," IEEE/OSA Journal of Optical Communications and Networking, Vol. 5, No. 10, pp. A46-A56 (2012).

[14]T. Tanimura, et al., "In-Band FSK Auxiliary Channel toward Adaptive Optical Network" IEICE Transactions (B), Vol. J96-B, No. 3, pp. 263-273 (2013).

[15] A. Caballero, et al., "Performance monitoring techniques supporting cognitive optical networking," IEEE International Conference on Transparent Optical Networks, Tu.B1.3. (2013).

[16] M. Ferraro et al., "Advanced digital waveforms for low-Earth-orbit (LEO) FSO links," Proc. SPIE, 112720A (2020).

[17] Y. Ou et al., " Demonstration of Virtualizeable and Software-Defined Optical Transceiver," IEEE J. Lightwave Tech., vol. 34, No.8, pp.1916-1924 (2015).

[18]K. Kikuchi, "Fundamentals of Coherent Optical Fiber Communications," IEEE J. Lightwave Tech., vol. 34, No.1, pp.157-179 (2016).

[19]F. N. Hauske, "Optical Performance Monitoring in Digital Coherent Receivers," IEEE J. Lightwave Tech., vol. 27, No.16, pp.3623-3631 (2009).

[20] Y. Tian, et al., "Inter-satellite Integrated Laser Communication/Ranging Link with Feedback-Homodyne Detection and Fractional Symbol Ranging," IEEE International Conference on Space Optical Systems and applications (ICSOS), S7.1 (2019). 
[21]Z. Tao, et al., "Transmission Characteristics Monitoring by Digital Signal Processing in Coherent Receiver," OptoElectronics and Communications Conference (OECC), 6B1-2 (2018).

[22] T. Anfray, et al., "Assessment of the performance of DPSK and OOK modulations at $25 \mathrm{~Gb} / \mathrm{s}$ for satellite-based optical communications," IEEE International Conference on Space Optical Systems and applications (ICSOS), S2.4 (2019).

[23] B. Barritt, et al., "Loon SDN: Applicability to NASA's next-generation space communications architecture," IEEE Aerospace Conference (2018).

[24]E. Leboffe et al., "Multi-mission capable $1550 \mathrm{~nm}$ lasercom terminal for space applications," Proc. SPIE, 1127205 (2020). 